\title{
BIOPHYSICS
}

Vol. 9, pp. 123-129 (2013)

doi: $10.2142 /$ biophysics.9.123

\section{Development of a rapid Buffer-exchange system for time-resolved ATR-FTIR spectroscopy with the step-scan mode}

\author{
Yuji Furutani ${ }^{1,2}$, Tetsunari Kimura ${ }^{1,2}$ and Kido Okamoto ${ }^{3}$ \\ ${ }^{1}$ Department of Life and Coordination-Complex Molecular Science, Institute for Molecular Science, 38 Nishigo-Naka, Myodaiji, Okazaki \\ 444-8585, Japan \\ ${ }^{2}$ Department of Structural Molecular Science, The Graduate University for Advanced Studies (SOKENDAI), 38 Nishigo-Naka, Myodaiji, \\ Okazaki 444-8585, Japan \\ ${ }^{3}$ UNISOKU Co., Ltd., 2-4-3 Kasugano, Hirakata, Osaka 573-0131, Japan
}

Received June 6, 2013; accepted July 16, 2013

\begin{abstract}
Attenuated total reflectance (ATR)-FTIR spectroscopy has been widely used to probe protein structural changes under various stimuli, such as light absorption, voltage change, and ligand binding, in aqueous conditions. Timeresolved measurements require a trigger, which can be controlled electronically; therefore, light and voltage changes are suitable. Here we developed a novel, rapid buffer-exchange system for time-resolved ATR-FTIR spectroscopy to monitor the ligand- or ion-binding reaction of a protein. By using the step-scan mode (time resolution; $2.5 \mathrm{~ms}$ ), we confirmed the completion of the buffer-exchange reaction within $\sim 25 \mathrm{~ms}$; the process was monitored by the infrared absorption change of a nitrate band at $1,350 \mathrm{~cm}^{-1}$. We also demonstrated the anionbinding reaction of a membrane protein, Natronomonas pharaonis halorhodopsin $(p H R)$, which binds a chloride ion in the initial anion-binding site near the retinal chromophore. The formation of chloride- or nitrate-bound $p H R$ was confirmed by an increase of the retinal absorption band at $1,528 \mathrm{~cm}^{-1}$. It also should be noted that low sample consumption ( $\sim 1 \mu \mathrm{g}$ of protein) makes this new method a powerful technique to understand ligandprotein and ion-protein interactions, particularly for membrane proteins.
\end{abstract}

Corresponding author: Yuji Furutani, Department of Life and Coordination-Complex Molecular Science, Institute for Molecular Science, 38 Nishigo-Naka, Myodaiji, Okazaki 444-8585, Japan. e-mail: furutani@ims.ac.jp
Key words: membrane protein, ion-protein interaction, halorhodopsin, attenuated total reflectance, stopped-flow technique

Elucidation of the structural changes of proteins in a time-resolved manner with sufficient time resolution is required for the better understanding of the molecular mechanisms of various proteins. Currently, optical microscopy of single molecules and high-speed atomic force microscopy are powerful methods for the real-time observation of the motion of proteins upon action ${ }^{1,2}$ Both of these methods visualize the movement, rotation, bending, and fluctuation of single protein molecules. However, the former method actually observes the motion of a probe, such as a fluorescent dye or a colloidal gold particle, and the latter method has limitations in monitoring the structural changes inside the protein. To understand the realization of protein motions, the knowledge of the protein structures in atomic detail and their dynamics in an appropriate time range with relation to their functions should be acquired. Spectroscopy has been widely used to achieve this goal.

Light-receptor proteins have a hallmark suitable for timeresolved spectroscopic measurements, because their functions are trigged by light, which enables us to monitor structural changes up to the femtosecond time scale by using an ultra-short laser pulse ${ }^{3}$. Redox proteins have been investigated by utilizing voltage change as a trigger ${ }^{4}$. While the time resolution of the voltage-triggering method is lower 
than that of the light-triggering method because of the generation of capacitative current in the system, it can achieve a time resolution of milliseconds ${ }^{5}$. On the other hand, although ligand- and ion-binding reactions are ubiquitous, triggering the reaction by ligand or ion binding has been limited. The limited use of this approach is due to its much lower time resolution than the other two methods and the practical difficulties in the physical replacement of the buffer solution in the system, such as vibrations and high inner pressure. These problems can be overcome by special techniques, such as the use of caged compounds, which is basically a light-triggering method; a continuous-flow technique $^{6,7}$; and a stopped-flow (STF) technique ${ }^{8,9}$ used in this study.

Attenuated total reflectance (ATR)-FTIR spectroscopy is a powerful technique for studying the structure of a membrane protein in a lipid bilayer immersed in an aqueous solution ${ }^{10}$. In particular, a difference spectrum calculated from the spectra recorded before and after providing various types of stimuli (e.g., light ${ }^{11}$, voltage change ${ }^{12}$, ligands ${ }^{13}$, and ions ${ }^{14}$ ) provides information about structural changes relating to the reactions of proteins. Another important advantage of ATR-FTIR is low sample consumption; only $\sim 1 \mu \mathrm{g}$ of the membrane protein reconstituted into a lipid bilayer is required. Light-induced- and voltage-change-induced difference ATR-FTIR spectroscopy has been applied to many membrane proteins, such as a microbial rhodopsin ${ }^{11}$, a photosynthetic reaction center ${ }^{15}$, and cytochrome $c$ oxidase $^{16}$. The reactions of these proteins were also analyzed by time-resolved methods with time resolution from the nano- to millisecond range. Perfusion-induced difference ATR-FTIR spectroscopy has also been developed to analyze ligand-protein and ion-protein interactions ${ }^{13,16}$. The time resolution of the perfusion-induced method depends on the time for exchanging a buffer solution in a sample chamber, which usually takes several minutes because of the slow flow rate of a perfusion peristaltic pump.

We developed a novel, rapid buffer-exchange system for time-resolved ATR-FTIR spectroscopy. The system was based on a commercially available STF system, which can supply a buffer solution to a sample chamber or an optical cell. In this method, a membrane protein sample was adsorbed on an ATR crystal, and the buffer solution above the sample was rapidly exchanged. The feasibility of its application to the ligand-binding reaction of a membrane protein was confirmed by the chloride- and nitrate-binding reactions of Natronomonas pharaonis halorhodopsin ( $p \mathrm{HR})$, which is a light-driven chloride-ion-pump protein ${ }^{17}$. The initial anion-binding site of $p \mathrm{HR}$ is near the retinal Schiff base and can bind not only a chloride ion but also a nitrate. A previous ATR-FTIR spectroscopy study of $p$ HR reported the chloride- and nitrate-binding-induced difference IR spectra; a retinal ethylenic band was observed at $1,525 \mathrm{~cm}^{-1}$ in the chloride-bound form and $1,529 \mathrm{~cm}^{-1}$ in the nitratebound form ${ }^{18}$. The increase in nitrate was also monitored by a nitrate absorption band at $1,350 \mathrm{~cm}^{-1}$. The FTIR method enabled us to simultaneously monitor infrared absorption at the frequencies of interest. Such a multiplex advantage improves the signal-to-noise $(\mathrm{S} / \mathrm{N})$ ratio more efficiently than dispersive spectrometry (the Fellgett advantage). The step-scan mode was applied for time-resolved ATR-FTIR measurements. The step-scan method has a higher time resolution (up to $10 \mathrm{~ns}$ in the specifications) than the conventional rapid-scan mode; however, the dead time of the exchange buffer is estimated to be tenths of a millisecond, and thus limits the effective time resolution. (We chose $2.5 \mathrm{~ms}$ to achieve the sufficient time resolution for our measurement system.)

\section{Materials and Methods}

\section{Sample preparation}

$p$ HR was expressed and purified as previously described with minor modification ${ }^{19}$. His-tag-purified $p H R$ was further purified using a Mono $Q$ anion-exchange column with an AKTA purifier (GE Healthcare). The ratio of the absorbance of $p \mathrm{HR}$ at 280 and $575 \mathrm{~nm}$ was below 1.8 after Mono Q column purification. The sample was reconstituted into egg PC liposomes (Sigma-Aldrich) with a lipid-to-protein molar ratio of 20:1 by adsorbing $n$-dodecyl- $\beta$-D-maltopyranoside (DDM) micelles on Bio-Beads (Bio-Rad) for about $12 \mathrm{~h}$. The final protein concentration was confirmed to be $\sim 8 \mu \mathrm{M}$, which was estimated from the absorbance at $578 \mathrm{~nm}(0.44$ O.D.) with an extinction coefficient $\left(\varepsilon_{578}\right)$ of $54,000 \mathrm{M}^{-1} \mathrm{~cm}^{-1}$.

\section{Design of the rapid buffer-exchange system}

In the supplementary material, the movie demonstrating the motion of the rapid buffer-exchange system is available on the website of BIOPHYSICS. The flow was induced by a pneumatic drive, which is a conventional system for STF applications. The length of the flow pulses was defined by a plunger-latch mechanism that allowed the syringe contents to be discharged as a fixed number of shots dependent on the pitch of the latching stops with the barrel. The pitch could be controlled by the motor drive from $0.1 \mathrm{~mm}$ to $7 \mathrm{~mm}$. We used a barrel with $2.5-\mathrm{mL}$ gas-tight syringes that had Teflon plungers. With this syringe, a $2.4-\mathrm{mm}$ pitch corresponded to a $0.1-\mathrm{mL}$ discharge of the solution. As the pneumatic drive ram operated, the motor drove the latching stop forward and retracted, ready for the next drive stroke. This "push" procedure could be alternately repeated for syringe 1 and 2 , or syringe 2 could be slowly pushed by the motor. We used the latter option for the step-scan method in this study. It takes about $1 \mathrm{~s}$ to wash out the buffer existing in the chamber by slowly pushing the syringe. The exchanging volume is $100 \mu \mathrm{L}$ which is same amount used for the rapid-buffer exchange process. The buffer is almost completely replaced by both of the processes. The refilling operation involved simply drawing back the plungers with the latching plates, which resulted in the refilling of the 
syringes. Then, the motor drive pushed up the latching plate to the initial shot position. The valve system was electrically interlocked to prevent attempts to refill when the flow system was directed through the ATR chamber. The $20-\mathrm{mm}$ drive ram was operated at $0.12 \mathrm{MPa}$, which theoretically produced a hydraulic pressure of $6 \mathrm{MPa}$ in the drive system. The actual pressure during the drive was likely less than the theoretical value, $6 \mathrm{MPa}$.

The system components that caused the most problems during development were the valves that permitted the refilling of the drive syringes with buffers. A valve constructed from blocks of Teflon and ceramics proved successful by clamping very tightly between the outer segments, while the rotation of the Teflon parts could still be attained by operating the pneumatic drive ram at $0.25 \mathrm{MPa}$.
The buffers were loaded into the sample chamber by PEEK tubes ( $1 \mathrm{~mm}$ internal diameter), and the buffers were ejected through the Teflon tube ( $1 \mathrm{~mm}$ internal diameter).

The system was developed by K. O. in UNISOKU based on the concept and ideas from Y.F. and T.K.

\section{Buffer-exchange system for ATR crystal plate}

Figure 1A shows the buffer-exchange assembly, while the inset of Figure 1B shows top and side views of the ATR chamber. Two concerns in the design were to maintain the dead volume as low as possible and to prevent another buffer from flowing into the chamber. In the DuraSamplIR ATR crystal, although its shape was circular, the IR light mainly passed through the center line of the crystal according to the beam path of the spectrometer. Therefore, the flow

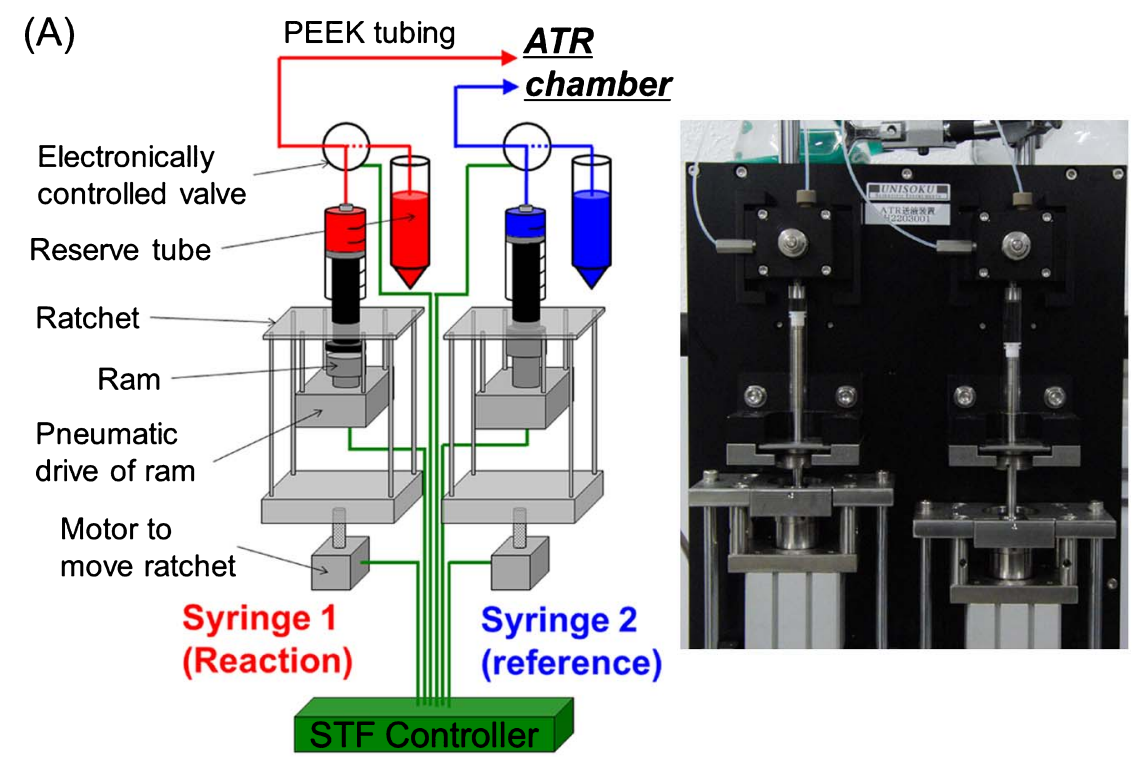

(B)

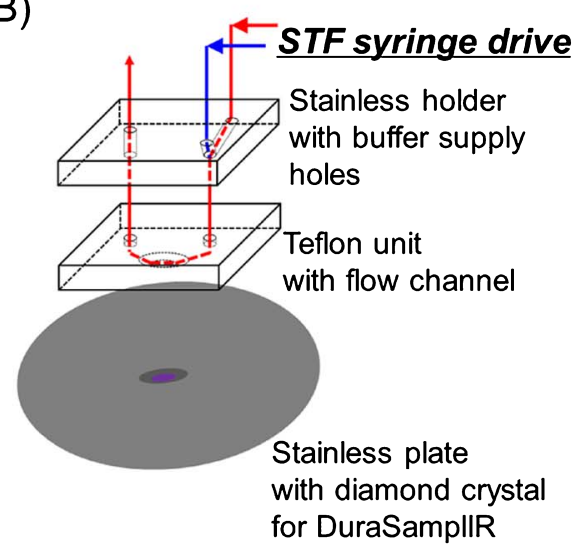

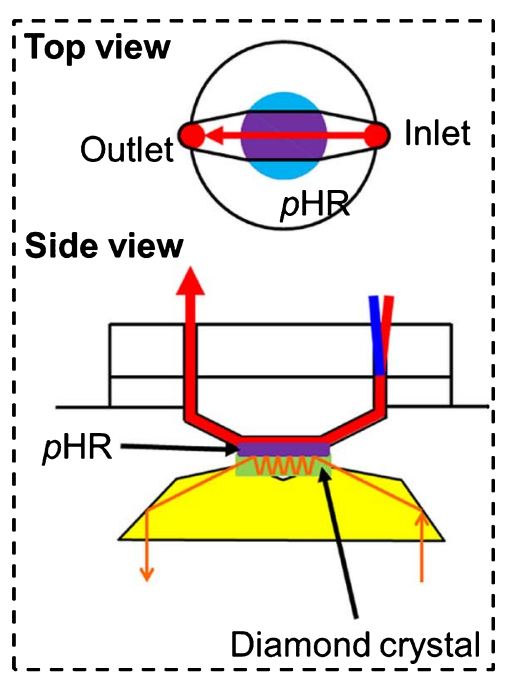

Figure 1 Schematic figures and pictures of the developed rapid buffer-exchange system. (A) The STF drive system was mounted on ground, while the spectrometer was placed on a bench which vibrationally isolated from ground. The only connection was the PEEK tubes that loaded the buffers into the ATR chamber. (B) The ATR chamber was mounted on the stainless plate with a diamond crystal in the DuraSamplIR II ATR accessory. The inset shows the top and side close-up views of the ATR chamber around the diamond crystal. 
channel did not have to cover the entire area of the ATR crystal. The Teflon unit ( $4 \mathrm{~mm}$ in thickness) with a flow channel ( $1 \mathrm{~mm}$ in depth and $2 \mathrm{~mm}$ in width) was mounted onto the ATR crystal ( $4 \mathrm{~mm}$ in diameter) and tightened by the stainless parts holding the flow channels to supply and eject the buffers. The Teflon-flow channel covered $61 \%$ of the area of the ATR crystal. The dead volume between the drive syringe and the chamber was $0.47 \mathrm{~mL}$ for each line, while that between the chamber and the sample space was $4 \mu \mathrm{L}$, and the sample space required $\sim 35 \mu \mathrm{L}$ of buffer. It is possible to reduce the chamber volume to be exchanged and the dead space prior to the chamber by using a narrower flow channel and a PEEK tube with a smaller internal diameter, but the pressure in the drive system could increase and cause problems with valve leakage, flow rate, and syringe breakage. Another important point that was considered was the vibration by the drive-pulse shock. Since the vibration critically affects the resulting spectrum in FTIR spectroscopy, it was important that the drive system was vibrationally isolated from the spectrometer. The connection was limited to the PEEK tubing, and the drive system was completely isolated from the shock-absorbing bench on which the spectrometer was placed. The chamber was tightened much more robustly than the standard buffer-perfusion system for the ATR-FTIR setup; a stainless plate with 12 bolts was used to clamp the Teflon assembly. The clamping bolts had to be tightened carefully and systematically by using a torque driver to prevent leakage.

\section{IR spectrometer and the achievable time resolution}

The STF system was interfaced with a Bruker Vertex 80 spectrometer. The step-scan mode was applied to measure with a time resolution of $2.5 \mathrm{~ms}$. The transient signals were measured at 333 sampling points with 2,000 time slices $(5 \mathrm{~s}$ in total) in the time-resolved step-scan mode, with a spectral resolution of $8 \mathrm{~cm}^{-1}$ and a spectral region from 1,900 to $988 \mathrm{~cm}^{-1}$, indicating that there were 333 total shots of the reaction buffer. Ten time slices were recorded before the buffer shot and used for a reference spectrum. Each interferogram was converted to a single-beam spectrum by Fourier transformation from 1,900 to $988 \mathrm{~cm}^{-1}$.

\section{Triggering and data acquisition}

The step-scan mode was operated as follows. The timing of the STF drive and data acquisition was controlled by the STF controller. The OPUS software running the step-scan measurement awaited the trigger from the controller in each mirror position. The triggering pulse to start the measurement was emitted from the STF controller and the measurement started. The delay of the STF drive was electronically controlled from $2 \mathrm{~ms}$ to $100 \mathrm{~ms}$, during which time the signals for the reference could be measured. After the set delay time, the STF syringe drive was turned out, and the signals from buffer exchange were measured. After the termination of the measurement, the interferometer mirror moved to the next sampling position, and the OPUS software again awaited the trigger from the STF controller. During the waiting time, the second buffer was drawn into the chamber to return the sample to its initial condition. The STF system was set to stay in the pushed position for at least $2 \mathrm{~s}$, because the immediate movement of the alternate syringe after the syringe drive induced the backflash of buffers or disturbed the stable measurements. The syringes were repeatedly filled every 17 shots when using a $100-\mu \mathrm{L}$ shot volume with a 2.5 $\mathrm{mL}$ syringe, as was programmed into the STF controller. Twenty refills were required to finish a step-scan measurement. A total of 340 shots were sufficient for obtaining data at 333 sampling points. It takes about an hour to complete one step-scan experiment.

\section{Buffer-exchange experiment}

The step-scan mode was tested by exchanging the buffer from pure water to a $50 \mathrm{mM} \mathrm{NaNO}$ solution. A step-scan measurement was measured with a time resolution of $2.5 \mathrm{~ms}$.

\section{Buffer-exchange experiment with a $p H R$ film}

A $10-\mu \mathrm{L}$ aliquot of the suspension containing $p \mathrm{HR}$ reconstituted into PC liposomes with 0.44 O.D. at $578 \mathrm{~nm}$ $(\sim 8 \mu \mathrm{M})$ was dried on the ATR crystal. The flow channel was established, and the dried $p H R$ film was rehydrated by introducing a buffer solution. The anion-binding reaction was triggered by buffer exchange to $200 \mathrm{mM}$ MOPS ( $\mathrm{pH}$ 7.0)-containing $20 \mathrm{mM} \mathrm{NaCl}$ or $\mathrm{NaNO}_{3}$. The MOPS buffer (200 mM, pH 7.0) without any salt was used for reference measurements. Three step-scan measurements (with a time resolution of $2.5 \mathrm{~ms}$ ) were averaged for each condition.

\section{Results and Discussion}

\section{Anion-binding process of $\boldsymbol{p H R}$}

$p \mathrm{HR}$ can bind $\mathrm{NO}_{3}^{-}$molecule near the protonated Schiff base linkage with the all-trans retinal chromophore, similar to the mechanism of chloride binding. The dissociation constant of $\mathrm{NO}_{3}^{-}$was estimated to be $11 \mathrm{mM}$, slightly higher than that of the chloride ion $(2 \mathrm{mM})$, in a lipid membrane ${ }^{17,20}$. Ion-binding events alter the visible absorption spectrum from the anion-free form $\left(\lambda_{\max }=599 \mathrm{~nm}\right)$ to the chloride-binding form $\left(\lambda_{\max }=577 \mathrm{~nm}\right)$ or the nitrate-binding form $\left(\lambda_{\max }=571 \mathrm{~nm}\right)^{17,19}$. The retinal vibrational modes are also affected by anion binding, and the $\mathrm{C}=\mathrm{C}$ stretching mode of the anion-free form at $1,511 \mathrm{~cm}^{-1}$ is shifted to that of the chloride-binding form at $1,525 \mathrm{~cm}^{-1}$ or the nitratebinding form at $1,529 \mathrm{~cm}^{-118}$. Therefore, the $1,529-\mathrm{cm}^{-1}$ band is a useful probe for monitoring the formation of nitratebound $p \mathrm{HR}$ in the rapid buffer-exchange experiment.

The suspension of $p \mathrm{HR}$ reconstituted into PC liposomes was dried on the ATR crystal and then repeatedly rehydrated by a buffer solution with and without $\mathrm{NO}_{3}^{-}$(Fig. 2A). The drying process made the sample adhere on the surface of the ATR crystal. After washing the sample using the per- 


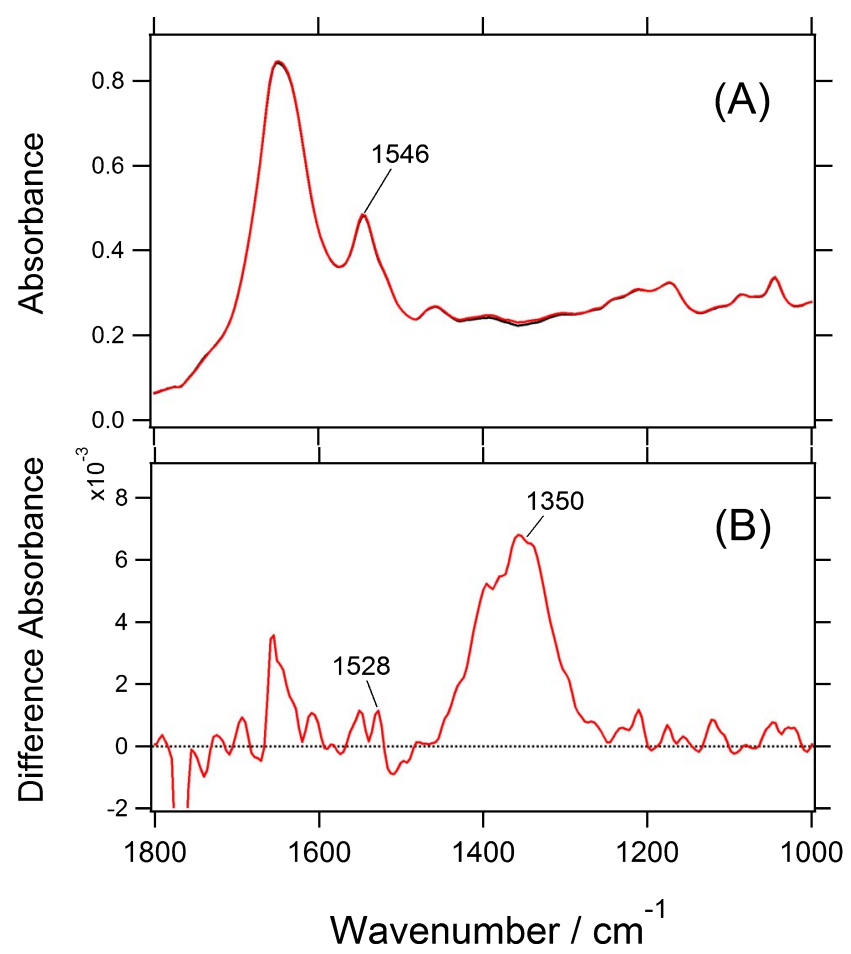

Figure 2 Absolute absorption spectra of $p H R$ immersed in $200 \mathrm{mM}$ MOPS buffer (pH 7.0) with (red line) or without (black line) $20 \mathrm{mM}$ $\mathrm{NaNO}_{3}(\mathrm{~A})$ and their difference spectrum (B).

fusion buffer-exchange system, the residual sample was tightly adsorbed and was not peeled off during the rapid buffer-exchange experiment with $\sim 5,000$ shots (Sup. Fig. 1). The static $\mathrm{NO}_{3}^{-}$binding-induced difference spectrum was corrected by exchanging the buffer solution with one containing $20 \mathrm{mM} \mathrm{NaNO}_{3}$ (Fig. 2B). The spectrum was very similar to that previously reported and showed a large positive peak at $1,350 \mathrm{~cm}^{-1}$, which is attributed to $\mathrm{NO}_{3}^{-}$in the chamber, and another positive peak was observed at $1,528 \mathrm{~cm}^{-1}$, which is attributed to the ethylenic stretching mode of the retinal chromophore of $\mathrm{NO}_{3}{ }^{-}$-bound $p \mathrm{HR}$.

Rapid buffer-exchange experiments with the step-scan mode were performed for the nitrate-binding reaction of $p H R$. The time-resolved spectra are shown in the logarithmic time scale (Fig. 3). The large peak from the NO stretching vibration was observed at $1,350 \mathrm{~cm}^{-1}$, and the peak from the $\mathrm{C}=\mathrm{C}$ stretching mode of the nitrate-bound form of the retinal chromophore was also observed at $1,528 \mathrm{~cm}^{-1}$. The perturbation induced at several other frequencies by the injection of the buffer solution was most clearly observed in the time region from $25 \mathrm{~ms}$ to $100 \mathrm{~ms}$, when the perturbation at 1,350 and $1,528 \mathrm{~cm}^{-1}$ was less significant.

The time courses of the difference in absorbance at 1,350 and $1,528 \mathrm{~cm}^{-1}$ are shown in Figures 4 and 5, respectively. The kinetics of the increase in $\mathrm{NO}_{3}^{-}$was very similar with and without the $p \mathrm{HR}$ sample, suggesting that $\mathrm{NO}_{3}{ }^{-}$permeated well into the $p \mathrm{HR}$ sample on the ATR crystal (Figs. 4A and 4B). The thickness of the sample affects the initial rise, because thicker sample requires longer time for permeation of solute into the stacks of the liposomes (Sup. Fig. 2). In the case of the bare ATR crystal, Figure 4B (dotted line) shows that the $\mathrm{NO}_{3}^{-}$absorbance increased within $\sim 25 \mathrm{~ms}$. The initial increase was fit to a single exponential curve with a time constant of $\sim 10 \mathrm{~ms}$. After the rapid increase in the $\mathrm{NO}_{3}^{-}$absorbance, a rapid decrease in the signal and recovery into the stable phase were observed. The reason

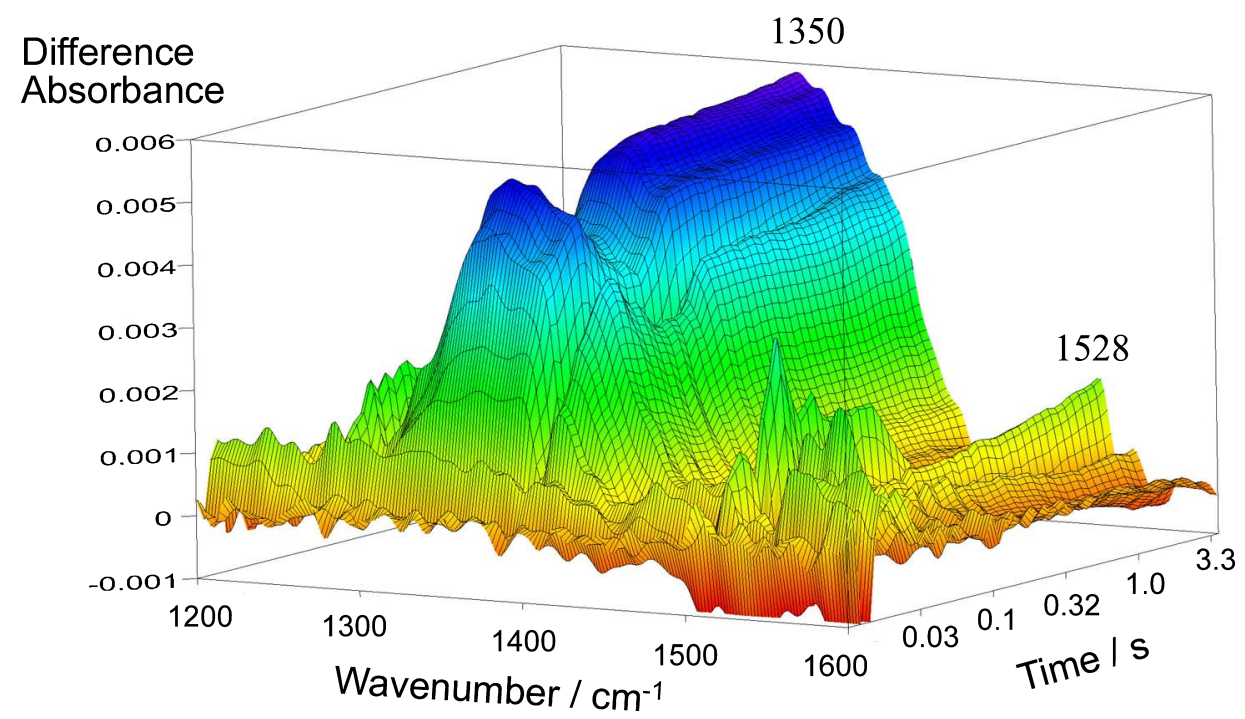

Figure 3 Time-resolved difference infrared spectra in the 1,600-1,200 $\mathrm{cm}^{-1}$ region, which were recorded by ATR-FTIR spectroscopy with the step-scan mode with a time resolution of $2.5 \mathrm{~ms}$. The spectra are an average of three measurements (total of 999 shots). The injection of nitrate was confirmed by a large absorbance change around $1,350 \mathrm{~cm}^{-1}$. Nitrate binding to $p \mathrm{HR}$ was monitored by an increase in the $\mathrm{C}=\mathrm{C}$ stretching mode at $1,528 \mathrm{~cm}^{-1}$. 


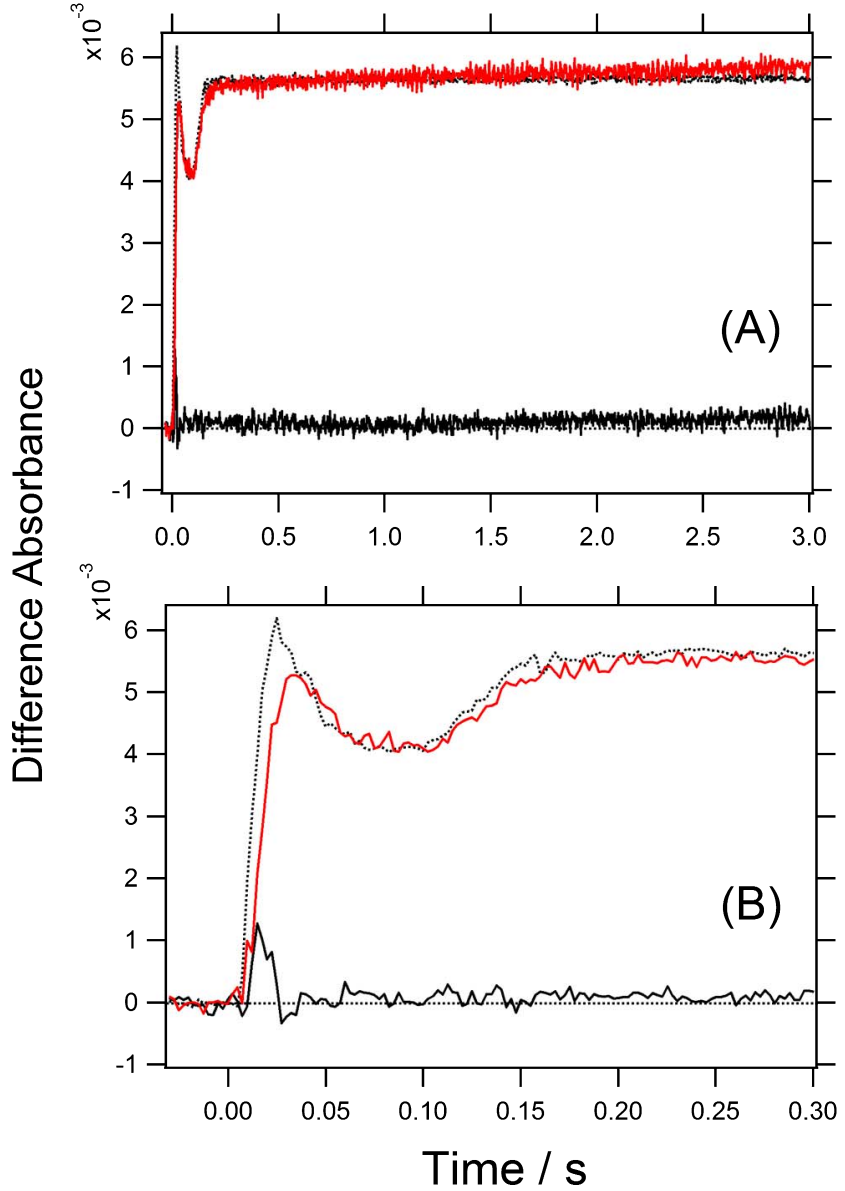

Figure 4 Rapid buffer exchange from $200 \mathrm{mM}$ MOPS (pH 7.0) to $200 \mathrm{mM}$ MOPS ( $\mathrm{pH} 7.0)+20 \mathrm{mM} \mathrm{NaNO}_{3}$ was monitored by the change in nitrate absorption at $1,350 \mathrm{~cm}^{-1}$ until $3.0 \mathrm{~s}(\mathrm{~A})$ and $0.3 \mathrm{~s}(\mathrm{~B})$. The traces in red were recorded with the $p \mathrm{HR}$ film sample, while the dottedline traces were recorded with the bare ATR crystal (from pure water to a $50 \mathrm{mM} \mathrm{NaNO}_{3}$ solution). The black line was obtained by the rapid buffer exchange from $200 \mathrm{mM}$ MOPS buffer to the same buffer to indicate baseline fluctuation.

for the spike in the initial phase is not clear. The peak did not originate from the injection shock, which was observed in the MOPS-only experiment (black trace). The chamber shape, pressure for pushing the syringe, and amount of buffer solution to be replaced did not affect the appearance of the initial phase. The dependence of the initial phase on the sample thickness was also investigated (Sup. Fig. 2). The spike was not observed in a thicker film, but the kinetics of buffer exchange slowed down.

The increase in absorbance at $1,528 \mathrm{~cm}^{-1}$ was plotted in Figure 5. The red and blue traces show the kinetics of binding with $\mathrm{NO}_{3}{ }^{-}$and $\mathrm{Cl}^{-}$, respectively. The black trace shows a baseline fluctuation, which was recorded with the injection of the same buffer as the washing buffer (200 mM MOPS). The increase in the signal with chloride binding is greater than that with nitrate binding, which corresponds well with the difference in the dissociation constants $\left(11 \mathrm{mM}\right.$ for $\mathrm{NO}_{3}^{-}$

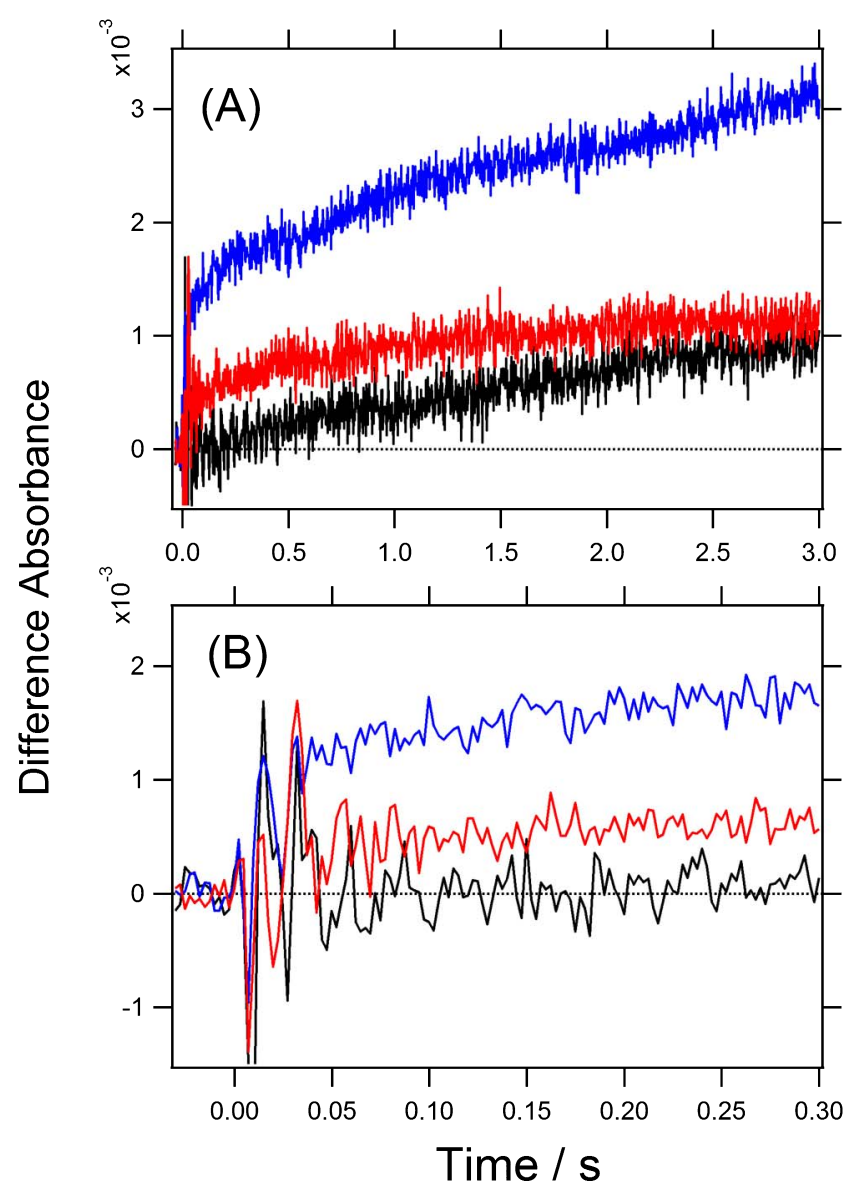

Figure 5 Absorbance change of the nitrate-binding reaction of $p \mathrm{HR}$ triggered by the rapid buffer exchange from $200 \mathrm{mM}$ MOPS (pH 7.0) to $200 \mathrm{mM}$ MOPS (pH 7.0) $+20 \mathrm{mM} \mathrm{NaNO}_{3}$ (red) or $200 \mathrm{mM}$ MOPS (pH 7.0) $+20 \mathrm{mM} \mathrm{NaCl}$ (blue). The time course of the retinal $\mathrm{C}=\mathrm{C}$ stretching vibrational mode of $p \mathrm{HR}$ at $1,528 \mathrm{~cm}^{-1}$ is shown until $3.0 \mathrm{~s}$ (A) and $0.3 \mathrm{~s}(\mathrm{~B})$. The time course at $1,528 \mathrm{~cm}^{-1}$ of the rapid buffer exchange from $200 \mathrm{mM}$ MOPS to the same buffer is shown in black.

and $2 \mathrm{mM}$ for $\mathrm{Cl}^{-}$).

A previous STF visible absorption experiment on $p \mathrm{HR}$ solubilized into DDM micelles revealed that the chloridebinding reaction exhibits a biphasic nature, with a faster $\left(\tau_{\text {fast }}=16 \mathrm{~ms}\right)$ and a slower phase $\left(\tau_{\text {slow }}=200 \mathrm{~ms}\right)^{19}$. The time trace for chloride binding in our measurement was fitted by two exponentials $\left(\tau_{1}=35 \mathrm{~ms}, \tau_{2}=1.3 \mathrm{~s}\right)$ after subtracting the baseline drift. The difference in kinetics may be explained by different sample conditions (in DDM micelles vs. PC liposomes). The faster phase may be difficult to measure by our system because of the dead time for the buffer-exchange reaction on the ATR crystal. An increase in the $\mathrm{S} / \mathrm{N}$ ratio by accumulating data and a longer observation time will give us more accurate results to describe the anion-binding reaction of $p \mathrm{HR}$ reconstituted into $\mathrm{PC}$ liposomes in the future. 


\section{Conclusion}

We developed a novel, rapid buffer-exchange apparatus for ATR-FTIR spectroscopy and applied it to the anionbinding reaction of $p \mathrm{HR}$. We confirmed that buffer exchange was completed within $\sim 25 \mathrm{~ms}$, and the anion-binding processes were followed by monitoring the retinal vibration. This method is applicable to many membrane proteins in lipid environments and requires low consumption of protein sample $(\sim 1 \mu \mathrm{g})$.

\section{Acknowledgements}

We thank the Equipment Development Center in the Institute for Molecular Science (IMS) for fabricating the buffer-exchange chamber, especially Mr. Masaki Aoyama and Ms. Noriko Takada. We also thank the Research Center of Integrative Molecular Systems (CIMoS) in the IMS. Prof. Makoto Demura provided the plasmid of $p$ HR. This work was partly supported by the Japan Society for the Promotion of Science (JSPS) KAKENHI to Y.F. (22770159) and T.K. (23687022). The authors would like to thank Enago (www.enago.jp) for the English language review.

\section{References}

1. Masaike, T., Koyama-Horibe, F., Oiwa, K., Yoshida, M. \& Nishizaka, T. Cooperative three-step motions in catalytic subunits of $F_{1}$-ATPase correlate with 80 degrees and 40 degrees substep rotations. Nat. Struct. Mol. Biol. 15, 1326-1333 (2008).

2. Uchihashi, T., Iino, R., Ando, T. \& Noji, H. High-speed atomic force microscopy reveals rotary catalysis of rotorless $\mathrm{F}_{1}$ ATPase. Science 333, 755-758 (2011).

3. Nakamura, T., Takeuchi, S., Shibata, M., Demura, M., Kandori, H. \& Tahara, T. Ultrafast pump-probe study of the primary photoreaction process in pharaonis halorhodopsin: Halide ion dependence and isomerization dynamics. J. Phys. Chem. B 112, 12795-12800 (2008).

4. Ataka, K. \& Heberle, J. Use of surface enhanced infrared absorption spectroscopy (SEIRA) to probe the functionality of a protein monolayer. Biopolymers 82, 415-419 (2006).

5. Osawa, M. Surface-enhanced infrared absorption. in NearField Optics and Surface Plasmon Polaritons. (Kawata, S. ed.) vol. 81, pp. 163-187 (Springer, Berlin Heidelberg, 2001).

6. Regenfuss, P., Clegg, R. M., Fulwyler, M. J., Barrantes, F. J. \& Jovin, T.M. Mixing liquids in microseconds. Rev. Sci. Instrum. 56, 283-290 (1985).

7. Kimura, T., Maeda, A., Nishiguchi, S., Ishimori, K., Morishima, I., Konno, T., Goto, Y. \& Takahashi, S. Dehydration of main- chain amides in the final folding step of single-chain monellin revealed by time-resolved infrared spectroscopy. Proc. Natl. Acad. Sci. USA 105, 13391-13396 (2008).

8. Chance, B. Rapid and sensitive spectrophotometry. I. The accelerated and stopped-flow methods for the measurement of the reaction kinetics and spectra of unstable compounds in the visible region of the spectrum. Rev. Sci. Instrum. 22, 619627 (1951).

9. Gibson, Q.H. \& Milnes, L. Apparatus for rapid and sensitive spectrophotometry. Biochem. J. 91, 161-171 (1964).

10. Nyquist, R. M., Ataka, K. \& Heberle, J. The molecular mechanism of membrane proteins probed by evanescent infrared waves. ChemBioChem 5, 431-436 (2004).

11. Kitade, Y., Furutani, Y., Kamo, N. \& Kandori, H. Proton release group of pharaonis phoborhodopsin revealed by ATRFTIR spectroscopy. Biochemistry 48, 1595-1603 (2009).

12. Jiang, X., Zaitseva, E., Schmidt, M., Siebert, F., Engelhard, M., Schlesinger, R., Ataka, K., Vogel, R. \& Heberle, J. Resolving voltage-dependent structural changes of a membrane photoreceptor by surface-enhanced IR difference spectroscopy. Proc. Natl. Acad. Sci. USA 105, 12113-12117 (2008).

13. Rich, P.R. \& Iwaki, M. Methods to probe protein transitions with ATR infrared spectroscopy. Mol. Biosyst. 3, 398-407 (2007).

14. Furutani, Y., Murata, T. \& Kandori, H. Sodium or lithium ionbinding-induced structural changes in the K-ring of V-ATPase from Enterococcus hirae revealed by ATR-FTIR spectroscopy. J. Am. Chem. Soc. 133, 2860-2863 (2011).

15. Okubo, T. \& Noguchi, T. Selective detection of the structural changes upon photoreactions of several redox cofactors in photosystem II by means of light-induced ATR-FTIR difference spectroscopy. Spectrochim. Acta, Part A 66, 863-868 (2007).

16. Nyquist, R.M., Heitbrink, D., Bolwien, C., Wells, T.A., Gennis, R. B. \& Heberle, J. Perfusion-induced redox differences in cytochrome c oxidase: ATR/FT-IR spectroscopy. FEBS Lett. 505, 63-67 (2001).

17. Váró, G., Brown, L. S., Sasaki, J., Kandori, H., Maeda, A., Needleman, R. \& Lanyi, J. K. Light-driven chloride-ion transport by halorhodopsin from Natronobacterium pharaonis. 1. The photochemical cycle. Biochemistry 34, 14490-14499 (1995).

18. Guijarro, J., Engelhard, M. \& Siebert, F. Anion uptake in halorhodopsin from Natromonas pharaonis studied by FTIR spectroscopy: Consequences for the anion transport mechanism. Biochemistry 45, 11578-11588 (2006).

19. Sato, M., Kanamori, T., Kamo, N., Demura, M. \& Nitta, K. Stopped-flow analysis on anion binding to blue-form halorhodopsin from Natronobacterium pharaonis: Comparison with the anion-uptake process during the photocycle. Biochemistry 41, 2452-2458 (2002).

20. Bálint, Z., Lakatos, M., Ganea, C., Lanyi, J.K. \& Váró, G. The nitrate transporting photochemical reaction cycle of the pharaonis halorhodopsin. Biophys. J. 86, 1655-1663 (2004). 